\title{
El emprendimiento como base en la condición socioeconómica
}

\section{Entrepreneurship as a basis in the socioeconomic condition}

Cristhian Andrés Sigüenza Aguirre

Universidad Técnica de Machala, Ecuador

Bernard Cesar Macías Sares, MG.

Universidad Técnica de Machala, Ecuador

Bill Jonathan Serrano Orellana, MAE

Universidad Técnica de Machala, Ecuador

Autor para correspondencia: casiguenza_est@utmachala.edu.ec

Fecha de recepción: 09 de Septiembre de 2016 - Fecha de aceptación: 10 de Diciembre de 2016

Resumen: Esta investigación determina los índices de la capacidad emprendedora que existen en los niveles socioeconómicos de los habitantes de la ciudad de Machala. Se analizó a la capacidad emprendedora a través de cinco dimensiones, para lo cual se aplicó una encuesta a una muestra de 982 personas. Se plantearon dos hipótesis que están relacionadas con la capacidad emprendedora para determinar su influencia en los niveles socioeconómicos. Para corroborar las hipótesis planteadas se implementó un modelo de regresión lineal (ANOVA), estableciendo como variable dependiente a la capacidad emprendedora y como variables independientes los niveles socioeconómicos que se aproxima mediante viables dicotómicas, donde la variable de comparación es el nivel socioeconómico bajo . El resultado reflejo que existe una diferencia significativa en la capacidad emprendedora respecto a los niveles socioeconómicos y llegando a la conclusión de que la capacidad emprendedora aumenta en función de los niveles socioeconómicos.

Palabras claves: emprendimiento; capacidad emprendedora; nivel socioeconómico

Abstract: This researching work is to demonstrate the enterprenourship abilities of people in the city of Machala, between different socioeconomic status. Enterprenuorship abilities were analized through five dimentions, on which 982 people were surveyed. Two enterprenuorship abilities related hypothesis were presented, in order to determine their influence in different social and economic levels. A linear regression model (ANOVA) was implented in order to prove the hypothesis. The enterprenuorship hability was stablished as a dependent variable, and as independants variables, the socioeconomic status was taken, and we had dicotomous aproaching, where the comparison variable in the low social and economic level. As a result we had that there is a big difference between the socioeconomic status and the enterprenourshp ability on low levels so, we may infer that the enterprenourship ability rises with the social and economic level.

Key words: entrepreneurship; entrepreneurship hability; socioeconomic status 


\section{Introducción}

El emprender ha venido teniendo mucho énfasis en los últimos años, y a medida que va captando más adeptos se vuelve un tema de mayor interés para la investigación, generando la interrogante de porqué las personas se ven en la necesidad de emprender, saliendo de su zona de confort para enfrentarse a nuevos retos. Sabiendo que el emprender es una tarea de valientes, se debe esclarecer cuales son los individuos que tienen el perfil para logar obtener ventaja de las oportunidades que el entorno les presenta.

La creciente tendencia de emprender ha marcado de manera significativa la economía de los países, logrando acaparando la atención de los diversos entes sociales ya que se ha convertido en una de las principales fuentes de crecimiento económico (Mancilla y Amorós, 2012), adicionalmente este fenómeno proporciona una mayor diversificación económica mejorando la liquidez a escala local (Kliksberg, 1999).

La iniciativa a emprender es un arte que se debe cultivar, fomentar e impulsar en las personas para lograr alcanzar resultados que permitan cuantificar los progresos económicos, los cuales han sido previstos obtener en el marco de un periodo económico (Sáenz y López, 2015), incentivando al individuo a crear su propia microempresa para lograr elevar las fuentes de empleo en la región como se ha podido evidenciar en los países desarrollados (Villardón, Alda, y Elexpuru, 2012).

Los individuos que cuentan con la capacidad emprendedora son elementos de gran aporte a los niveles socioeconómicos (Bradley y Corwyn, 2002). Sin embargo Rodríguez (2004), dice que el aspecto socioeconómico es un factor que no ha sido considerado como una variable mayor relevancia en investigaciones anteriores.

Las investigaciones del Global Entrepreneurship Monitor (GEM) han considerado al género, la edad y el nivel de escolaridad como variables para determinar que individuo cuenta con la capacidad emprendedora (Amorós, Guerra, \& Carrillo, 2008). Dando origen al objetivo de la investigación ¿en qué nivel socioeconómico logramos encontrar un mayor indicé de la capacidad emprendedora?

La capacidad emprendedora en la actualidad es considerada una virtud muy codiciada, ya que según Villa (2008) los emprendedores son los generadores del progreso en la sociedad, por su aporte en el incremento de los niveles de productividad.

El estudio se encaminara en determinar qué nivel socioeconómico cuenta con una mayor capacidad emprendedora, permitiéndole aprovechar las oportunidades del mercado.

Implementando un instrumento de medición se determinará si existen diferencias significativas entre los niveles socioeconómicos.

\section{Marco Teórico}

En investigaciones primarias al emprendedor se le dio el calificativo de ser una persona ecuánime el mismo que entre sus prioridades, busca incrementar de manera significativa sus 
réditos económicos (Rusque, 2005), asumiendo con responsabilidad los riesgos que esto le pudiera ocasionar de manera directa a su capital social (Fernández, Alegre, y Chiva, 2012), y en el ascenso empresarial debe fortalecer sus habilidades y destrezas. (Betancourt, 2004).

Para crear el vínculo entre el emprendimiento y la condición socioeconómico, es necesario conocer que emprendimiento cuenta con el nuevo esquema empresarial (Santos, Barroso, y Guzmán, 2013), generando impacto en la comunidad, creando compromisos de responsabilidad, perseverancia y mejora continua (Duarte y Ruiz, 2009). Otorgándole al individuo el seudónimo de emprendedor porque logra ofrecer nuevos productos y servicios en el mercado (González, Arrizabalaga, Navarro, y Peña, 2009).

A un empresario exitoso se lo logra distinguir fácilmente de entre quienes se mantienen en una zona de confort, ya que entre sus cualidades más importantes se destaca su habilidad para encontrar nuevas oportunidades y sus dotes de negociante eficiente (Espíritu, 2011),cumpliendo de manera responsable con sus obligaciones que se contrajeran en el libre ejercicio de la profesión, muy abiertamente de calificar a una persona por sus acciones, en el ámbito comercial se lo juzga por quien es, lo que permite formar un criterio solido haciéndolo acreedor a un respeto inmensurable, y en base a todo lo antes expuesto, se concibe una reputación que difícilmente a verse manchada a lo largo de su trayectoria empresarial (Sobrado y Fernández, 2010).

Cualquier persona puede emprender, pero eso no les garantiza que logren alcanzar un rotundo éxito (Trujillo, Girola, Figeralla, y Navas, 2002), es necesario realizar una distinción entre los que cuentan con la capacidad emprendedora y quienes simplemente lo hacen porque la necesidad los conlleva a buscar una nueva forma de generar ingresos (Sanabria y Burgos, 2004). Las personas que nacen con una inclinación para emprender se los logra diferenciar de las personas comunes (Cabana, Cortes, Plaza, Castillo, y Álvarez, 2013), porque mantienen claro su objetivo y buscan marcar la diferencia en la sociedad (Vargas, 2001).

La capacidad emprendedora se forja a temprana edad, relacionándose de manera directa con el entorno que los rodea, los niveles de aceptación del individuo en la sociedad se encuentran estrechamente vinculados con la relación intrafamiliar (Carlos, Contreras, Silva, y Liquidano, 2015), determinando que la iniciativa emprendedora la podemos encontrar con mayor frecuencia en los senos familiares, que cuentan con una experiencia previa en empezar un nuevo ente económico (Delgado, Goméz, Romero, y Vázquezi, 2008). Recalcando que la capacidad emprendedora está directamente relacionado con los valores culturales y los estudios sociodemográficos dejan en evidencia que las personas que tienen un conocimiento previo de los riesgos posmaterialistas cuentan con una mayor inclinación a ser personas capaces de emprender, porque mantienen un estatus económico por encima del nivel común (Pinillos Costa, 2011).

En lo que va del siglo XXI se han realizado importantes investigaciones, que determinan un mayor grado de involucramiento de las mujeres en temas de emprendimiento, sin desmerecer la participación de los hombres en temas relacionados (Díaz, Hernández, Sánchez, y Postigo, 2010).La capacidad emprendedora es un llamado de alerta para la generación de nuevas fuentes de empleados, llegando a la conclusión de que quienes emprenden, son aquellas personas que a lo largo de su vida han ido acumulando experiencias y conocimientos en ramas específicas, 
creando una tendencia en el cual el emprender depende de la edad que oscila entre los 37 y 53 años (López, Romero, y Díaz, 2012). Y se evidencio que la condición económica no ha sido considerada como una variable al momento de determinar quiénes son las personas que disponen de una mayor capacidad emprendedora (Hinojosa y Albornoz, 2013).

Martín Cruz, Gómez Barahona, \& Rodríguez Escudero (2015), en su estudio de la capacidad emprendedora consideran como variables de gran relevancia a los rasgos como son: la edad, los niveles de educación, el género y la experiencia previa, dejando sin efecto los niveles socioeconómicos, en el cual se centra esta investigación y dejando abierta la brecha de que investigaciones anteriores no le han dado la mayor importancia a esta condición de vital relevancia.

Geográficamente Ecuador está ubicado en una región predominada por el subdesarrollo, generando una diferencia significativa en los niveles socioeconómicos (Amorós, 2011). Se vive una etapa económicamente agónica, donde los ingresos promedios que mantiene una familia común están por debajo la canasta básica, obligando a que se generen nuevas fuentes de ingresos (Bruneau y Machado, 2006).

Segovia y Melgarejo (2011), nos dicen que a inicios del siglo XX las familias optaron por crear empresas unipersonales para poder generar ingresionas adicionales, sin embargo Cornella (2007) y el Centro de innovación y desarrollo empresarial (2007), argumentan que en la actualidad el concepto de empresas unipersonales ha sido modificado por empresas familiares, manteniendo el objetivo de obtener mayores ingresos.

\section{Metodología}

La obtención de datos fue mediante fuente primaria a través de un cuestionario aplicado a 982 individuos de la ciudad de Machala de un total de 276.669 habitantes según los datos que reposan en los archivos del INEC. Se inició con una investigación bibliográfica para determinar las variables del estudio (capacidad emprendedora y nivel socioeconómico). Luego se determinó la capacidad emprendedora con un instrumentos validado por Tinoco (2008) compuesto por cinco dimensiones ( conocimiento de sí mismo, visión del futuro, motivación de logro, planificación y persuasión) donde 0 es equivalente a nunca, 1 a casi nunca, 2 en ocasiones, 3 con frecuencia, 4 a casi siempre y 5 a siempre, mientras que para el nivel socioeconómico se recurrió al instrumento del INEC y los rangos de calificación son desde 0 a 1.000, como se puede evidenciar en la tabla 1.

Tabla 1.- Escala de los niveles socioeconómicos

\begin{tabular}{cc}
\hline Grupos Socioeconómicos & Puntaje \\
\hline Alto & De 846 a 1.000 puntos \\
Medio Alto & De 697 a 845 puntos \\
Medio Típico & De 536 a 696 puntos \\
Medio Bajo & De 317 a 535 puntos \\
Bajo & De 0 a 316 puntos \\
\hline
\end{tabular}

Fuente: INEC 
Fue necesario establecer una escala de calificación del instrumento para que determinar en qué rango se encuentra cada nivel socioeconómico, y de esta forma poder realizar una comparación entre las mismas.

Tabla 2.- Escala de calificación del instrumento

\begin{tabular}{ll}
\hline Puntaje & Calificación \\
\hline De 1 a 4 & Muy Bajo \\
De 5 a 8 & Bajo \\
De 9 a 12 & Mediano \\
De 13 a 16 & Alto \\
De 17 a 20 & Muy alto \\
\hline
\end{tabular}

Fuente: (Tinoco, 2008)

H1: En la ciudad de Machala el nivel socioeconómico determina la capacidad emprendedora de los habitantes.

H2: En las dimensiones de "conocimiento de sí mismo, visión de futuro, motivación de logro, planificación persuasión" se logran evidenciar diferencias de gran impacto entre los niveles socioeconómicos.

En esta investigación se implementó un modelo de regresión lineal (ANOVA), estableciendo como variable dependiente a la capacidad emprendedora de naturaleza cuantitativa y como variable independiente el nivel socioeconómico que se aproxima mediante viables dicotómicas, donde la variable de comparación es el nivel socioeconómico bajo.

\section{Resultados}

En este estudio, y con relación a las cualidades psicométricas del test construido para medir de forma directa la variable capacidad emprendedora e indirecta la variable de los niveles socioeconómicos, se lo efectuó de la siguiente manera:

Los resultados demostraron que existen diferencias significativas en la capacidad emprendedora en base a los niveles socioeconómicos, también se puede evidenciar que la capacidad emprendedora aumenta dependiendo del nivel socioeconómico en el que se encuentra el individuo. Los individuos del nivel socioeconómico bajo tienen menos capacidad emprendedora que los que se encuentran en un nivel socioeconómico superior, lo cual comprueba la hipótesis planteada. Como se lo demuestra en la tabla 3.

Tabla 3.- Puntaje obtenido según el nivel socioeconómico

\begin{tabular}{lccc}
\hline & Coeficiente & t & F \\
\hline 1.Capacidad Emprendedora & & \\
Medio Bajo & $16.49179^{* *}$ & 5.12 \\
Medito Típico & $24.07657^{* *}$ & 7.70 \\
Medio Alto & $25.66742^{* *}$ & 8.17 \\
Alto & $28.32653^{* *}$ & 8.45 \\
Constante & $52.57143^{* *}$ & 17.29 \\
$\quad$ Índices & & \\
\hline
\end{tabular}




\begin{tabular}{lcc}
\hline R-squared & 0.1119 & \\
$\mathrm{~F}(4,977)$ & $30.78 * *$ \\
\hline
\end{tabular}

Fuente: Elaboración propia

Tabla 4.- Puntaje total obtenido según el área de evaluación

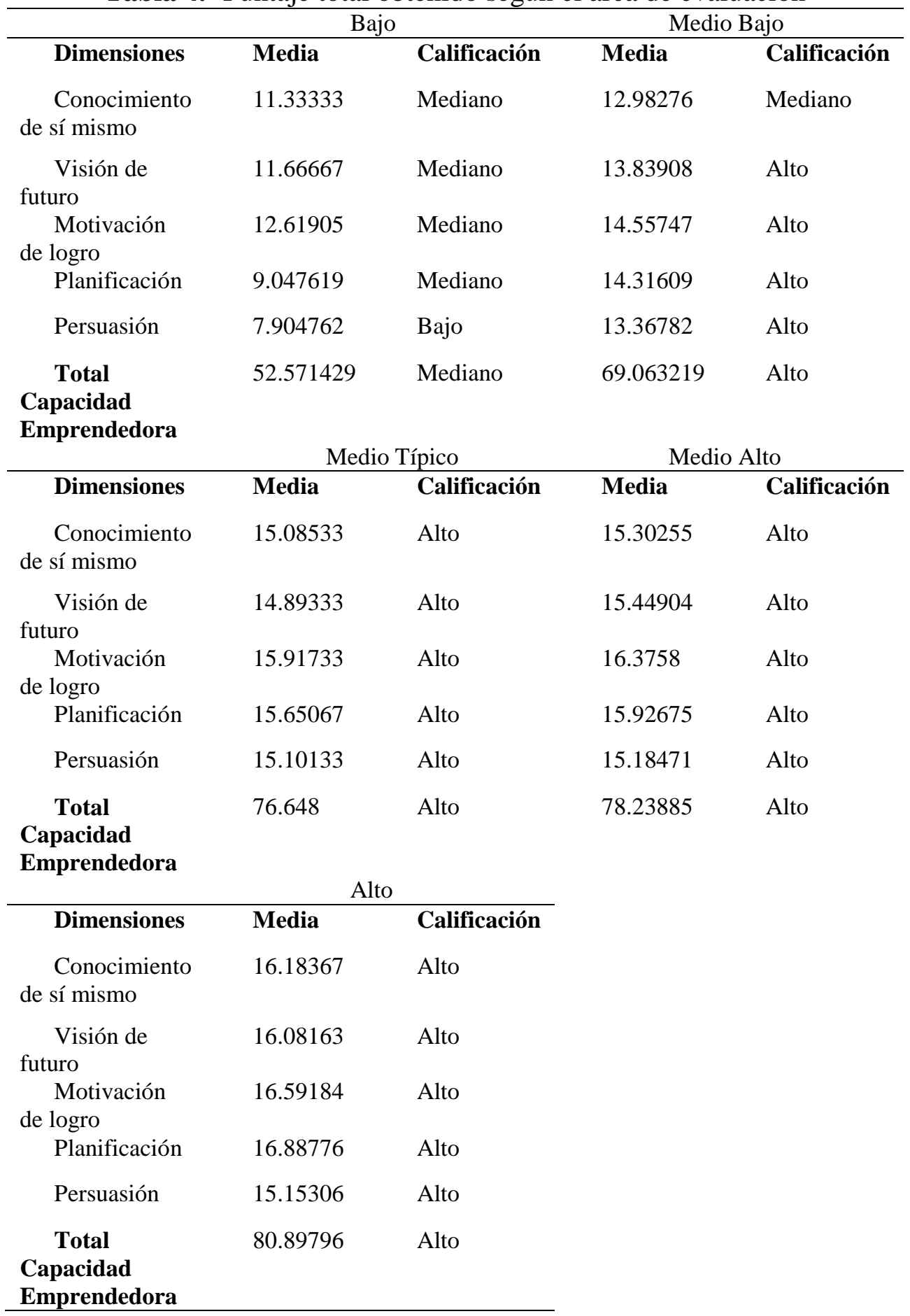

Fuente: Elaboración propia 
Los resultados de las dimensiones de la capacidad emprendedora, demostraron que a medida que los niveles socioeconómicos aumentan se obtiene una puntación más alta, con una leve variación en lo que respecta con la persuasión, considerando que el puntaje más alto lo encontramos en el nivel socioeconómico medio alto.

\section{Conclusiones}

En esta investigación se ha realizado un análisis del emprendimiento en base a la condición socioeconómica de los habitantes de la ciudad de Machala, considerando que las dimensiones que abarca a la capacidad emprendedora son: el conocimiento de sí mismo, la visión de futuro, motivación de logro, la planeación y la persuasión, se planteó un enfoque más detallado y exhaustivo con los niveles socioeconómicos establecidos los cuales son: bajo, medio bajo, medio típico, medio alto y alto.

Los resultados que se derivaron del análisis nos demostraron que existe una diferencia significativa, que se encuentra relacionada directamente con el nivel socioeconómico de los habitantes. En particular se logró constatar que las personas que pertenecen a la clase social baja, tiene la capacidad emprendedora más baja, lo que nos demuestra que con muy poca probabilidad se verá una iniciativa de emprendimiento de su parte.

La capacidad emprendedora, tiene una curva creciente a medida que existe un mejor nivel socioeconómico, siendo la clase social alta la que muestra los índices de la capacidad emprendedora más elevada, y con una mayor intencionalidad de emprender una de las ramas de sector económico.

En definitiva la capacidad emprendedora en los habitantes de la ciudad de Machala se la logra encontrar con mayor frecuencia en las personas que pertenecen al nivel socioeconómico alto, generando una mayor probabilidad de generación de emprendimientos, cuyos rasgos psicológicos como son el conocimiento de sí mismo, la visión del futuro, la motivación de logro, la planificación y la persuasión, pudieron haber sido transmitidos en base a los antecedentes empresariales que tuvieron sus familiares o aun cierta experiencia previa en el emprendimiento.

\section{Bibliografía}

Alcaraz, R. E. (2004). Efectividad del Curso de Emprendedores, en el desarrollo del perfil emprendedor del alumno. RIEEE, 1-27.

Amorós, J. E. (2011). El proyecto global entrepreneurship monitor (GEM): una aproximación desde el contexto latinoamericano. Academia. Revista latinoamericana de administración (46), 1-15.

Amorós, J. E., Guerra, M., \& Carrillo, J. M. (2008). Reporte Nacional de Chile 2008. Global Entrepreneurship Monitor, 1(1), 1-95.

Betancourt, B. (2004). La capacidad emprendedora y el desarrollo de nuevas empresas. Cuadernos de administración, 20(32), 109-131. 
Bradley, R. H., \& Corwyn, R. F. (2002). Socioeconomic Status And Child Development. Annu. Rev. Psychol, (53), 371-399.

Bruneau, J., \& Machado, H. V. (2006). Empreendedorismo nos países de América Latina basado nos indicadores do Global Entrepreneurship Monitor (GEM). Panorama socioeconómico, 24(33).

Cabana, R., Cortes, I., Plaza, D., Castillo, M., \& Álvarez, A. (2013). Análisis de las capacidades emprendedoras potenciales y efectivas en alumnos de centros de educación superior. Journal of Technology Management \& Innovation, 8(1), 65 - 75.

Carlos, C. E., Contreras, L. E., Silva, M. d., \& Liquidano, M. d. (2015). El Espíritu Emprendedor y un Factor que Influencia su Desarrollo Temprano. Conciencia Tecnológica, enero-junio (49), 46-51.

Centro de innovación y desarrollo empresarial. (2007). La gestión de la innovación en la empresa. Revista de contabilidad y dirección, 6, 55-64.

Cornella, A. (2007). Pensar desde la innovación. Revista de contabilidad y dirección, 6, 13-35.

Delgado, M. I., Gómez, L., Romero, A. M., \& Vázquezi, E. (2008). Determinantes sociales y cognitivos en el espíritu emprendedor: un estudio exploratorio entre. Cuadernos de Gestión, 8(1), 11-24.

Díaz, J. C., Hernández, R., Sánchez, M. d., \& Postigo, M. V. (2010). Actividad Emprendedora y género. Un estudio comparativo. Revista Europea de dirección y economía de la empresa., 19(2), 83-98.

Duarte, T., \& Ruiz, M. (2009). Emprendimiento, una opción para el desarrollo. Scientia Et Technica, 15(43), 326-331.

Espíritu, R. (2011). Análisis de la intención emprendedora en estudiantes universitarios a través de los rasgos de. Multiciencias, 4(1), 65-75.

Fernández, A., Alegre, J., \& Chiva, R. (2012). Orientación emprendedora, capacidad de aprendizaje organizativo y desempeño innovador. Journal of technology management \& innovation, 7(2), 157-170.

González, J. L., Arrizabalaga, A. M., Navarro, M. N., \& Peña, I. (2009). Estudio sobre la capacidad de innovacion y actividad emprendedora en el ámbito sub-regional: El caso de la comunidad autónoma del país Vasco. Investigaciones Regionales (15), 55-87.

Hinojosa, S., \& Albornoz, C. (2013). Ganas de emprender y felicidad: un estudio exploratorio a partir del global entrepreneurship monitor en Chile. Journal of Technology Management \& Innovation, 8(1), 76-89. 
Kliksberg, B. (1999). Capital social y cultura, clases esenciales del desarrollo. Revista de la Cepal 69, 85-102.

López, M., Romero, P. M., \& Díaz, R. (2012). Motivaciones para emprender: un análisis de diferencias entre hombres y mujeres. Economía Industrial (383), 75-84.

Mancilla, C., \& Amorós, J. E. (2012). La influencia de factores socio-culturales en el emprendimiento, evidencia en CHile 2007-2010. Multidisciplinary Business Review, 5(1), $14-25$.

Pinillos Costa, M. J. (2011). Cultura postmaterialista y variación en el espíritu emprendedor. Investigaciones Europeas de dirección y economía de las empresas, 17(1), 37-55.

Rusque, A. M. (2005). Capacidad Emprendedora y capital social. Revista Venezolana de Análisis de Conyuntura, 6(2), 189-202.

Sáenz, N., \& López, A. L. (2015). Las Competencias De Emprendimiento Social,Coems: Aproximación A Través De Programas De Formación Universitaria En Iberoamérica. Revesco(119), 159-182.

Sanabria, N. J., \& Burgos, A. (2004). Competencia, empresa y espíritu emprendedor. Escuela de Administración de Negocios, 4(52), 59-67.

Santos, F., Barroso, M., \& Guzmán, C. (2013). La economía global y los emprendimiento sociales. Revista de Economía Mundial (35), 177-196.

Segovia, A., \& Melgarejo, Z. (2011). La famiempresa, un sistema vivo y abierto de desarrollo socioeconómico. Tendencias, 12(2), 181-199.

Sobrado, L., \& Fernández, E. (2010). Competencias emprendedoras y desarrollo del espíritu emprendedor en los centros educativos. Educación XX1, 13(1), 15-38.

Tinoco, O. (2008). Medición de la capacidad emprendedora de ingresantes a la facultad de ingeniería industrial de la UNMSM. Industrial Data, 11(2), 18-23.

Trujillo, E., Girola, A., Figeralla, X., \& Navas, E. (2002). Diseño de material multimedia interactivo orientado al estímulo de la capacidad emprendedora. Anales, 2(2), 33-50.

Vargas, A. (2001). El cooperativismo como fórmula empresarial de futuro para canalizar el espíritu emprendedor colectivo. Galega de Economía, 10(1), 1-15.

Villa, M. (2008). El espíritu emprendedor, la gran fuerza de progreso. Cuadernos de pensamiento político, 2(18), 215-232. 
Villardón, L., Alda, R., \& Elexpuru, I. (2012). Propuesto y validación de un perfil de competencias de la persona emprendedora. Implicaciones para la formación. Electronic Journal of Research in Educational Psychology, 10(28), 1057-1080. 